\title{
Electromagnetic Fluctuations in an Equilibrium Plasma ${ }^{1}$
}

\author{
Ronald E. Burgess
}

\section{Contribution From the Department of Physics, University of British Columbia, Vancouver, Canada}

\author{
(Received October 22, 1964)
}

\begin{abstract}
The fluctuations in an electron-ion plasma in thermal equilibrium are first treated for the collisionless case. Separate consideration is given to the contributions of the transverse and longitudinal modes, and two approaches are used; one in terms of the energy density of the thermally excited plasma, the other in terms of the permittivity $\epsilon(K, \omega)$ appropriate to each mode. Some of the results have complete thermodynamic generality while some are based on the statistics of the plasma. Simple thermodynamic arguments are used to derive the radiation resistance of short electric and magnetic dipoles in a collisionless plasma. The relation between the spatial correlation properties of the longitudinal mode and Debye-Hückel theory is indicated. It is shown that in a plasma with collisions the fiuctuation temperature is equal to the common electron-ion temperature for any degree of degeneracy and any velocity dependence of relaxation time.
\end{abstract}

\section{Introduction}

In an earlier paper [Burgess, 1941] ${ }^{2}$ the author derived the electromagnetic field fluctuations of cavity radiation in a lossless nondispersive medium from the blackbody radiation energy density, and showed that an electric dipole immersed in the radiation would obey Nyquist's theorem.

These results are now extended to a more general medium, specifically a plasma, which has a complex and dispersive permittivity and can support both transverse and longitudinal waves. The transverse mode, characterized by $E$ and $H$ being perpendicular to the wave vector $K$, was the only one involved in the earlier treatment. The longitudinal mode in which $E$ and $K$ are parallel and $H$ is absent is accompanied by space charge density waves. In both modes damping occurs, even in the absence of collisions, by virtue of the Landau mechanism.

Since the dispersion equations are different for the two modes they will be considered separately; the thermal equilibrium energy density is calculated from the frequency-dependent permittivity and resolved into components arising from the fields and from the kinetic and potential energies of the particles.

We are concerned throughout with an unbounded equilibrium plasma which is therefore spatially homogeneous and statistically stationary. It is also assumed

${ }^{1}$ The research for this paper was supported by the Defence Research Board of Canada under Grant No. 9512-22.

${ }^{2}$ Burgess, R. E. (1941), Noise in receiving aerial systems, Proc. Phys. Soc. 53, 293-304 to be nonrelativistic; i.e., its temperature $T$ is smalk compared with $\mathrm{mc}^{2} / k$. Usually the ionic contribution to the fluctuations can be ignored if the ion mass is large compared with the electron mass but in the lowfrequency spectrum for the longitudinal mode the ionic and electronic terms become equal.

The spectral decomposition of a disturbance in the plasma is expressed in terms of the angular frequency $\omega$ and the wave number $K$; the linear response function of the plasma is then specified by the permittivity $\epsilon(K, \omega)$ which has different forms for the transverse and longitudinal modes. The presence of $K$ in the permittivity represents the effect of spatial dispersion; an excellent discussion of the electrodynamics of plasmas involving both frequency dispersion and spatial dispersion is provided by the monograph of Silin and Rukhadze [1961]. ${ }^{3}$

Fluctuation spectra are derived using the stochastic current density given by the fluctuation-dissipation theorem applied to the imaginary part of the permittivity.

Since in a vacuum cavity the electromagnetic field fluctuations are not zero it is necessary to demonstrate that the permittivity approach yields the correct result in the limit of $\operatorname{Im}(\epsilon)=0$.

For a system in equilibrium, any temperature which can be used to characterize the properties must equal the plasma temperature; thus, for instance the temperatures attributable to the electrons, the ions, and the electromagnetic field are all equal. Nevertheless erroneous statements have appeared in the literature

${ }^{3}$ Silin, V. P., and A. A. Rukhadze (1961), Elektromagnitnie Svoistva Plazmy i Plazmopodobnykh Sred. (Moscow, Gosatomizdat). 
to the effect that the fluctuation temperature of a plasma is equal to the electron temperature only in special circumstances e.g., a Maxwellian velocity distribution or a collision relaxation time independent of velocity. However in fact the equilibrium distribution (Fermi-Dirac) for the electrons and ions suffices to ensure identity of these temperatures independently of degeneracy or of any velocity dependence of collision frequency.

The temperature $T$ enters the expressions derived in this paper through the fluctuation-dissipation theorem. Thus at frequencies small compared with $k T / h$ the factor $k T$ appears while at higher frequencies the more general form $\hbar \omega(\exp \hbar \omega / k T-1)^{-1}$ is appropriate. For simplicity the $k T$ factor will be used except where it is desired to integrate over all frequencies a spectrum which would otherwise yield a divergent result. In deriving the radiation resistance of a dipole immersed in a plasma it is of course immaterial which form is used since the factor cancels in the comparison of mutually consistent expressions of Nyquist's relation and of the energy density.

Throughout we shall use rationalized MKS units. Frequency spectra will be defined with respect to $\omega$ and exist for positive values; thus, for a fluctuating quantity $X$

$$
\int_{0}^{\infty} \bar{X}_{\omega}^{2} d \omega=\bar{X}^{2}
$$

\section{Transverse Mode}

\subsection{Energy-Density Approach for the Transverse Mode}

In the transverse mode the electric and magnetic fields are perpendicular to the wave vector and no alternating component of space charge density occurs.

The number of modes per unit volume in the wave number interval $d K$ is

$$
d M=\left(k T K^{2} / \pi^{2}\right) d K
$$

taking into account two independent states of polarization. The mean energy density thus has the spectrum

$$
U=\left(k T K^{2} / \pi^{2}\right)(d K / d \omega) .
$$

It will here be assumed that the damping is sufficiently small for $\epsilon$ and $K$ to be substantially real for real $\omega$. It should be remembered that except for a few special functional forms of $\operatorname{Re} \epsilon(\omega)$ the imaginary part of $\epsilon(\omega)$ would not necessarily be negligible at all frequencies. The requirement of causality imposes restrictions on the behavior of $\epsilon(\omega)$ in the complex $\omega$-plane; one aspect of these is the Kramers-Kronig relations which connect $\operatorname{Re} \epsilon$ and $\operatorname{Im} \epsilon$.

Consider now a collision-free plasma for which the transverse permittivity is given by

$$
\epsilon / \epsilon_{0}=1-\omega_{p}^{2} / \omega^{2}
$$

where $\omega_{p}^{2}=n_{0} e^{2} / m \epsilon_{0}$. This familiar result is a good approximation for wave numbers $K$ small compared with the reciprocal of the Debye length $D=$ $\left(2 n_{0} e^{2} / \epsilon_{0} k T\right)^{1 / 2}$. In this case the Landau damping becomes very small and we write

$$
\begin{gathered}
K / \omega=\left(\mu_{0} \epsilon\right)^{1 / 2}=\left(1-\omega_{p}^{2} / \omega^{2}\right)^{1 / 2} / c \\
d k / d \omega=\epsilon_{0}\left(\mu_{0} / \epsilon\right)^{1 / 2}=\left(1-\omega_{p}^{2} / \omega^{2}\right)^{-1 / 2} / c .
\end{gathered}
$$

The mean square electric and magnetic fields are found from the relation

$$
\frac{1}{2} \epsilon \overline{E_{\omega}^{2}}=\frac{1}{2} \mu_{0} \overline{H_{\omega}^{2}}
$$

derived from Maxwell's equations and from the electric and magnetic energy densities

$$
U_{\omega}^{\mathrm{el}}=\frac{1}{2} \epsilon_{0} \overline{E_{\omega}^{2}} \quad U_{\omega}^{\mathrm{mag}}=\frac{1}{2} \mu_{0} \overline{H_{\omega}^{2}}
$$

while the kinetic energy density is

$$
U_{\omega}^{\mathrm{kin}}=\frac{1}{2} n_{0} m\left(\mathrm{e}^{2} \overline{E_{\omega}^{2}} / m^{2} \omega^{2}\right)=\frac{1}{2}\left(\omega_{p}^{2} / \omega^{2}\right) \epsilon_{0} \overline{E_{\omega}^{2}} .
$$

The total energy density is thus

$$
U_{\omega}=\epsilon_{0} \overline{E_{\omega}^{2}} .
$$

We note that the effective permittivity is $d(\omega \epsilon) / d \omega$ for the nonmagnetic energy, since the sum of the electric and kinetic energy densities can be written as

$$
U_{\omega}^{\mathrm{el}}+U_{\omega}^{\mathrm{kin}}=\frac{1}{2} \overline{E_{\omega}^{2}}(\epsilon+\omega d \epsilon / d \omega)
$$

Finally, therefore the field fluctuation spectra of the total field component perpendicular to a given wave vector $K$ are

$$
\overline{E_{\omega}^{2}}=k T \omega^{2} \mu_{0}^{3 / 2} \epsilon^{1 / 2} / \pi^{2}=k T \omega^{2}\left(1-\omega_{p}^{2} / \omega^{2}\right)^{1 / 2} / \pi^{2} c^{3} \epsilon_{0}
$$

$\overline{H_{\omega}^{2}}=k T \omega^{2} \mu_{0}^{1 / 2} \epsilon^{3 / 2} / \pi^{2}=k T \omega^{2}\left(1-\omega_{p}^{2} / \omega^{2}\right)^{3 / 2} / \pi^{2} c^{3} \mu_{0}$.

The following relation between the three energy components exists

$$
U_{\omega}^{\mathrm{el}}=U_{\omega}^{\mathrm{mag}}+U_{\omega}^{\mathrm{kin}}=\frac{1}{2} U_{\omega} .
$$

The total energy density spectrum has the frequency dependence

$$
U_{\omega}=\left(k T / \pi^{2} c^{3}\right) \omega^{2}\left(1-\omega_{p}^{2} / \omega^{2}\right)^{1 / 2}
$$

for $\omega>\omega_{p}$. 
If it is desired to integrate over all frequencies, $k T$ must be replaced by $\hbar \omega(\exp \hbar \omega / k T-1)^{-1}$ giving

$$
U=\frac{\hbar}{\pi^{2} c^{3}} \int \frac{\infty \omega^{2}\left(\omega^{2}-\omega_{p}^{2}\right)^{1 / 2}}{\omega_{p} \exp (\hbar \omega / k T)-1} d \omega
$$

As the plasma density is reduced to zero $\left(\omega_{p} \rightarrow 0\right)$ this integral increases to its maximum value given by the vacuum energy density of thermal radiation:

$$
U_{\mathrm{vac}}=\pi^{2} k^{4} T^{4} / 15 c^{3} \hbar^{3} .
$$

The fluctuation fields given above correspond to the resultant values. Thus the components in any direction have mean square values equal to one-third of the given expressions. The mean square emf induced in a short electric dipole of effective length $l$ is thus

$$
\overline{e ๘}=l^{2} k T \omega^{2} \mu_{0}^{3 / 2} \epsilon^{1 / 2} / 3 \pi^{2} .
$$

This must equal $(2 / \pi) k T R_{t}$ as required by Nyquist's theorem, where $R_{t}$ is the radiation resistance associated with the transverse mode. Thus

$$
R_{t}^{\mathrm{el}}=l^{2} \omega^{2} \mu_{0}^{3 / 2} \epsilon^{1 / 2} / 6 \pi .
$$

Similarly a short magnetic dipole formed by a planar loop of area $\boldsymbol{A}$ has a mean square induced emf.

$$
\overline{e_{\omega^{\prime}}^{2}}=\frac{1}{3}\left(\omega \mu_{0} A\right)^{2} \overline{H_{\omega}^{2}}=\frac{1}{3} K^{2} A^{2} \overline{E_{\omega^{\prime}}^{2}} .
$$

The radiation resistance for the magnetic dipole associated with transverse waves is thus

$$
R_{t}^{\operatorname{mag}}=A^{2} \omega^{4} \mu_{0}^{5 / 2} e^{3 / 2} / 6 \pi .
$$

For both the electric and magnetic dipoles the radiation resistance is reduced by an ambient plasma compared with vacuum, the factors being respectively $\left(\epsilon / \epsilon_{0}\right)^{1 / 2}$ and $\left(\epsilon / \epsilon_{0}\right)^{3 / 2}$.

This approach may also be extended to determining the spatial correlation of the field components. The cosine of the phase difference of the waves at two points $(1,2)$ separated by distance $r$ is averaged uniformly over all directions of arrival and all polarizations and gives

$$
\overline{E\left(r_{1}\right) E^{*}\left(r_{2}\right)_{\omega}} / \overline{E_{\omega}^{2}}=(\sin K r) / K r
$$

for the spatial autocorrelation coefficient appropriate to waves of wave number $K=\omega\left(\mu_{0} \epsilon\right)^{1 / 2}$. Thus

$$
\begin{aligned}
\overline{E\left(r_{1}\right) E^{*}\left(r_{2}\right)_{\omega}} & =\left(k T \omega^{2} \mu_{0}^{3 / 2} \epsilon^{1 / 2} / \pi^{2}\right)(\sin K r / K r) \\
& =\left(k T \omega \mu_{0} / \pi^{2}\right) \sin \left[\omega\left(\mu_{0} \epsilon\right)^{1 / 2} r\right] / r .
\end{aligned}
$$

This result will be compared later with that derived using the $\epsilon(K, \omega)$ method which includes the specific form of the imaginary part corresponding to Landau damping in a collisionless plasma.

\subsection{Permittivity Approach for the Transverse Mode}

In this approach the permittivity is taken to be a function of wave number as well as frequency in order to recognize the effects of spatial dispersion. For transverse waves the $\epsilon(K, \omega)$ so defined determines the dispersion equation by the relation [Silin and Rukhadze, 1961, eq (6.8)].

$$
\epsilon(K, \omega) / \epsilon_{0}=K^{2} c^{2} / \omega^{2} .
$$

For a Maxwellian equilibrium plasma with equal density of electrons and (singly charged) ions the explicit form of the permittivity in the absence of collisions is [Silin and Rukhadze, 1961, eq (14.3)].

$$
\begin{aligned}
\epsilon(K, \omega) / \epsilon_{0}=1 & -\left(\omega_{p}^{2} / \omega^{2}\right)\left[F(q)+i \pi^{1 / 2} q e^{-q^{2}}\right] \\
& -\left(\Omega_{p}^{2} / \omega^{2}\right)\left[F(Q)+i \pi^{1 / 2} Q e^{-Q^{2}}\right]
\end{aligned}
$$

where

$$
q^{2}=\omega^{2} m / 2 k T K^{2} \quad Q^{2}=\omega^{2} M / 2 k T K^{2}
$$

while

$$
F(x)=2 X e^{-x^{2}} \int_{0}^{x} e^{y^{2}} d y .
$$

For small values of $X, F(X) \simeq 2 X^{2}-4 X^{4} / 3+$. . for large values of $X, F(X) \sim 1+1 / 2 X^{2}+3 / 4 X^{4}+\ldots$

When we are considering results obtained from the explicit form of $\epsilon(K, \omega)$ we will usually be dealing with the case of large $q$ where the imaginary part (Landau damping) will be relatively small and to a close approximation

$$
\begin{aligned}
& \operatorname{Re} \epsilon(K, \omega) / \epsilon_{0} \simeq 1-\left(\omega_{p}^{2} / \omega^{2}\right)\left(1+k T K^{2} / \omega^{2} m\right) \\
&-\left(\Omega_{p}^{2} / \omega^{2}\right)\left(1+k T K^{2} / \omega^{2} M\right) .
\end{aligned}
$$

The dispersion equation then becomes on further approximation

$$
\omega^{2} \simeq \omega_{p}^{2}+\Omega_{p}^{2}+K^{2}\left(c^{2}+k T / m\right) \simeq \omega_{p}^{2}+K^{2} c^{2}
$$

in view of the assumptions of large ion/electron mass ratio $M / m$ and of a nonrelativistic plasma.

The fluctuation current density normal to a given wave vector $K$ has a spectral intensity determined by the plasma conductivity

$$
\overline{J_{K \omega}^{2}}=2(2 k T / \pi)(-\omega \operatorname{Im} \epsilon) .
$$

From Maxwell's equations the fluctuation field spectrun are

$$
\begin{aligned}
& \overline{E_{K \omega}^{2}}=(4 k T / \pi \omega) \operatorname{Im}\left[1 /\left(\epsilon-K^{2} / \omega^{2} \mu_{0}\right)\right] \\
& \overline{H_{K \omega}^{2}}=\left(4 k T K^{2} / \pi \omega^{3} \mu_{0}^{2}\right) \operatorname{Im}\left[1 /\left(\epsilon-K^{2} / \omega^{2} \mu_{0}\right)\right] .
\end{aligned}
$$


These expressions refer to the resultant quantity perpendicular to the wave vector and so give the sum of two independent orthogonal components. The $K \omega$ spectra may be integrated over $K$ (or $\omega$ ) to yield spectra in terms of $\omega$ (or $K$ ) and so give the purely temporal (or spatial) correlation functions. Performing these integrations invokes various sum rules which are listed in appendix 1 and which may be derived by contour integration. First, to find $J_{K}^{2}$ we integrate the conductivity of the Maxwellian plasma

$\sigma(K, \omega)=-\omega \operatorname{Im} \epsilon(K, \omega)=\pi^{1 / 2} \epsilon_{0} \omega^{-1}\left(\omega_{p}^{2} q e^{-q^{2}}\right.$
$\left.+\Omega_{p}^{2} Q e^{-Q^{2}}\right)$

for which

$$
\int_{0}^{\infty} \sigma(K, \omega) d \omega=\left(\pi \epsilon_{0} / 2\right)\left(\omega_{p}^{2}+\Omega_{p}^{2}\right) .
$$

Thence

$$
\overline{J_{K}^{2}}=2 n_{0} e^{2}(k T / m+k T / M) .
$$

This expression may be derived directly by considering the diffusion of the electrons and ions in the classical plasma.

Of relevance in considering the relaxation process in a collisionless plasma is the autocorrelation of the current density:

$$
\begin{aligned}
& \overline{J_{K}\left(t_{0}\right) J_{K}\left(t_{0}+t\right)} \\
& =2 k T n_{0} e^{2}\left[m^{-1} \exp \left(-K^{2} t^{2} k T / 2 m\right)\right. \\
& \left.+M^{-1} \exp \left(-K^{2} t^{2} k T / 2 M\right)\right] .
\end{aligned}
$$

This exhibits a gaussian autocorrelation function for the electrons and ions with respective relaxation times $\left(m / k T K^{2}\right)^{1 / 2}$ and $\left(M / k T K^{2}\right)^{1 / 2}$ corresponding to the gaussian thermal velocities of the particles.

The electric and magnetic field components are found using the results (A2) and (A3) for integration over frequency

$$
\begin{gathered}
\overline{E_{K}^{2}}=2 k T / \epsilon(K, \infty)=2 k T / \epsilon_{0} \\
\overline{H_{K}^{2}}=2 k T / \mu_{0} .
\end{gathered}
$$

Integration over wave number to obtain the frequency spectra can be readily carried through if we ignore the dependence of $\epsilon(K, \omega)$ on $K$ when $\epsilon$ appears in the form $\left(\epsilon-K^{2} / \omega^{2} \mu_{0}\right)$. This procedure is seen to be justified at small $K$ (large $q$ ) since then we are neglecting $k T^{\prime} / m$ by comparison with $c^{2}$. On the other hand at large $K($ small $Q$ ) the contributions to the real and imaginary parts of $\epsilon$ vary as $1 / K$ and are negligible compared with the $K^{2} / \omega^{2} \mu_{0}$ term.

Thus taking $\epsilon$ as a function only of $\omega$ we find an integration over $K$ space using integral (A8)

$$
\overline{E_{\omega}\left(r_{1}\right) E_{\omega}^{*}\left(r_{2}\right)}=\left(k T \omega \mu_{0} / \pi^{2}\right) e^{-\alpha r}(\sin \beta r) / r
$$

where

$$
\beta-i \alpha=\omega\left(\mu_{0} \epsilon\right)^{1 / 2} .
$$

This result may be compared with the expression (2.10) for a lossless plasma; it has in addition an attenuation factor arising from the imaginary part of $\epsilon$ (damping), while the $(\sin \beta r) / r$ factor due to the phase difference at the two observing points corresponds to a wave number $\omega \mu_{0}^{1 / 2} \operatorname{Re}\left(\epsilon^{1 / 2}\right)$.

It is equivalent to the form given by Silin and Rukhadze [1961] in the second term of their equation (9.41).

When the separation $r$ goes to zero the total electric field fluctuation spectrum

$$
\overline{E_{\omega}^{2}}=k T \omega^{2} \mu_{0}^{3 / 2} \operatorname{Re}\left(\epsilon^{1 / 2}\right) / \pi^{2} .
$$

When this is compared with the previous result (2.5) for a loss-free plasma it is seen that the appropriate generalization to a medium with damping $(\operatorname{Im} \epsilon \neq 0)$ is to replace $\epsilon^{1 / 2}$ by $\operatorname{Re}\left(\epsilon^{1 / 2}\right)$.

The electric field spectra derived in this section had their origin in the current density $J$ which is determined by $\operatorname{Im}(-\epsilon)$. However it is seen that as $\operatorname{Im}(-\epsilon)$ tends to zero the electric field spectrum and its spatial correlation do not vanish but tend to the value obtained by considering the electromagnetic energy density in a cavity filled with a lossless medium.

\section{Longitudinal Mode}

\subsection{Energy-Density Approach for the Longitudinal Mode}

The mode density per unit volume for the longitudinal mode is one-half of the expression used for the transverse mode because of the occurrence of only the single direction of the electric vector parallel to the wave vector. Thus the energy density spectrum is

$$
U_{\omega}=\left(k T K^{2} / 2 \pi^{2}\right)(d K / d \omega) .
$$

The electric field may be derived from a potential and there is no associated magnetic field. The energy density comprises the contributions of the electric field and the kinetic and potential energies of the electrons, and we ignore the ionic contribution as being negligible when the mass ratio $M / m$ is large:

$$
\left.U_{\omega}=\frac{1}{2} \epsilon_{0} \overline{E_{\omega}^{2}}+\frac{1}{2} n_{0} m \overline{u_{\omega}^{2}}+\frac{1}{2} n_{0} m u_{\theta}^{2} \overline{n_{\omega}^{2}} / n_{0}^{2}\right)
$$

where $u_{\omega}^{\overline{2}}$ is the spectrum of the electron velocity wave motion, $u_{\theta}^{2}$ is the mean square thermal velocity $3 \mathrm{kT} / \mathrm{m}$, and $n_{\omega}^{\overline{2}}$ is the spectrum of the electron density wave about the mean value $n_{0}$. The last term gives the contribution of the compressional energy density of the electron gas; since the pressure variations are given by

$$
p_{\omega}=\gamma n_{\omega} k T
$$


and the compressibility by

$$
\chi=1 / \gamma n_{0} k T
$$

the density of the compressional energy is

$$
\frac{1}{2} \overline{p_{\omega}^{2}} \chi=\frac{1}{2} \gamma k T\left(\overline{n_{\omega}^{2}} / n_{0}\right)
$$

and for longitudinal adiabatic compression we put $\gamma=3$, yielding the result given above.

By considering a longitudinal wave of the form $\exp i(\omega t-K r)$, we can write the continuity equation

$$
-i K n_{0} u_{\omega}+i \omega n_{\omega}=0
$$

and the equation of motion for unit volume as

$$
i \omega m n_{0} u_{\omega}=-n_{0} e E_{\omega}+i K m u_{\theta}^{2} n_{\omega}
$$

where the last term arises from the gradiant of the alternating pressure $\gamma n_{\omega} k T$.

Thus

$$
\omega n_{\omega} / K n_{0}=u_{\omega}=i e E_{\omega} / m \omega\left(1-u_{\theta}^{2} K^{2} / \omega^{2}\right) .
$$

The total energy density is then

$$
U_{\omega}=\frac{1}{2} \epsilon_{0} \overline{E_{\omega}^{2}}\left[1+\omega_{p}^{2}\left(\omega^{2}+u_{\theta}^{2} K^{2}\right) /\left(\omega^{2}-u_{\theta}^{2} K^{2}\right)^{2}\right] .
$$

Introducing now the dispersion equation for longitudinal waves in its first approximation, for wave numbers small compared with the reciprocal of the Debye radius:

$$
\omega^{2}=\omega_{p}^{2}+K^{2} u_{\theta}^{2}
$$

yields the results

$$
\begin{aligned}
U_{\omega} & =\left(\omega^{2} / \omega_{p}^{2}\right) \epsilon_{0} \overline{E_{\omega}^{2}} \\
U_{\omega}^{\mathrm{kin}} & =U_{\omega}^{\mathrm{pot}}+U_{\omega}^{\mathrm{el}}=\frac{1}{2} U_{\omega} .
\end{aligned}
$$

The reciprocals of the phase and group velocities are

$$
\begin{aligned}
K / \omega & =\left(1-\omega_{p}^{2} / \omega^{2}\right)^{1 / 2} / u_{\theta} \\
d K / d \omega & =\left(1-\omega_{p}^{2} / \omega^{2}\right)^{-1 / 2} / u_{\theta}
\end{aligned}
$$

leading to the result

$$
\overline{E_{\omega}^{2}}=k T \omega_{p}^{2}\left(1-\omega_{p}^{2} / \omega^{2}\right)^{1 / 2} / 2 \pi^{2} \epsilon_{0} u_{\theta}^{3} .
$$

This is larger than the electric field spectrum associated with the transverse mode by the factor

$$
\frac{1}{2}\left(\omega_{p}^{2} / \omega^{2}\right)\left(c^{3} / u_{\theta}^{3}\right)
$$

which may typically be very large compared with unity.

The radiation resistance of a short electric dipole, of effective length $l$, associated with the longitudinal mode is scaled by the same factor and thus

$$
R_{\text {long }}^{\mathrm{el}}=l^{2} \omega_{p}^{2} \epsilon^{1 / 2} / 12 \pi u_{\theta}^{3} \epsilon_{0}^{3 / 2} .
$$

\subsection{Permittivity Approach for the Longitudinal Mode}

Again the properties of the longitudinal mode are represented by permittivity $\epsilon(K, \omega)$. The dispersion equation for this mode is

$$
\epsilon(K, \omega)=0 .
$$

For a Maxwellian plasma without collisions the permittivity assumes the form [Silin and Rukhadze, 1961, eq (14.2)]

$$
\begin{aligned}
\epsilon(K, \omega) / \epsilon_{0}=1 & -\left(\omega_{p}^{2} / \omega^{2}\right) 2 q^{2}\left[F(q)-1+i \pi^{1 / 2} q e^{-q^{2}}\right] \\
& -\left(\Omega_{p}^{2} / \omega^{2}\right) 2 Q^{2}\left[F(Q)-1+i \pi^{1 / 2} Q e^{-Q^{2}}\right]
\end{aligned}
$$

where $q, Q$, and $F$ are as defined previously for the transverse mode. The factors in front of the square bracket terms have the common value

$$
\left(\omega_{p}^{2} / \omega^{2}\right) 2 q^{2}=\left(\Omega_{p}^{2} / \omega^{2}\right) 2 Q^{2}=n_{0} e^{2} / k T \epsilon_{0} K^{2}=1 / 2 K^{2} D^{2}
$$

where

$$
D=\left(k T \epsilon_{0} / 2 n_{0} e^{2}\right)^{1 / 2}
$$

is the Debye shielding radius.

For large values of $q(K D \ll 1)$ the asymptotic form of the permittivity on the assumption of $M \ll m$ is found to be

$$
\epsilon(K, \omega) / \epsilon_{0} \sim 1-\left(\omega_{p}^{2} / \omega^{2}\right)\left(1+3 / 2 q^{2}\right) .
$$

The corresponding dispersion equation takes on the well-known form appropriate to small wave numbers:

$$
\omega^{2}=\omega_{p}^{2}+(3 k T / m) K^{2} .
$$

The single Cartesian component of current density parallel to the wave vector has the spectrum

$$
\overline{J_{K \omega}^{2}}=(2 k T / \pi)(-\omega I m \epsilon) .
$$

Integrating over frequency gives

$$
\overline{J_{K}^{2}}=k T \epsilon_{0}\left(\omega_{p}^{2}+\Omega_{p}^{2}\right)=n_{0} e^{2}(k T / m+k T / M)
$$

which is one-half of the value associated with the transverse mode because of the two orthogonal components associated with the latter. Naturally the stochastic current density which excites the plasma will have 
three equal orthogonal components which cannot a priori be associated with any particular mode.

This current density is related by the wave relations to the other fluctuating quantities of interest namely: $E$, the longitudinal electric field; $\phi$, the electric potential, $\rho$ the space charge density. For a wave of the form exp $i(\omega t-K r)$ the relations are

$$
\begin{array}{ll}
E=J / i \omega \epsilon & \overline{E_{K \omega}^{2}}=\overline{J_{K \omega}^{2}} / \omega^{2}|\epsilon|^{2} \\
\phi=E / i K & \overline{\phi_{K \omega}^{2}}=\overline{J_{K \omega}^{2}} / K^{2} \omega^{2}|\epsilon|^{2} \\
\rho=-i K \epsilon_{0} E & \overline{\rho_{K \omega}^{2}}=\overline{J_{K \omega}^{2}} K^{2} \epsilon_{0}^{2} / \omega^{2}|\epsilon|^{2}
\end{array}
$$

so enabling the spectra of $E, \phi$, and $\rho$ to be related to that of $J$.

The electric field spectrum

$$
\overline{E_{K \omega}^{2}}=(2 k T / \pi \omega) \operatorname{Im}(1 / \epsilon)
$$

can be immediately integrated over all frequencies using (Al) to give

$$
\overline{E_{K}^{2}}=k T[1 / \epsilon(K, \infty)-1 / \epsilon(K, 0)] .
$$

Now $\epsilon(K, \infty)=\epsilon_{0}$ and $\epsilon(K, 0)=\epsilon_{0}\left(1+1 / K^{2} D^{2}\right)$

whence

$$
\overline{E_{K}^{2}}=k T / \epsilon_{0}\left(1+K^{2} D^{2}\right)
$$

In the evaluation of the static (zero frequency) permittivity $\epsilon(K, 0)$ it is seen from (3.5) at $q=Q=0$ that the ionic contribution is equal to the electronic contribution, and that the masses do not enter. In most other situations the large ratio $M / m$ results in the ionic contribution to the permittivity being negligible; this is the case when $q$ and $Q$ are large compared with unity, which implies a wave number small compared with $1 / D$.

The coulomb interaction in the ion-electron plasma vitally affects the electric field fluctuations in the longitudinal mode and this manifests itself through the appearance of the Debye distance providing a reference length for the wave number $K$ and for the spatial correlation. Integration over $K$ gives

$$
\overline{E\left(r_{1}\right) E\left(r_{2}\right)}=\left(k T / 4 \pi \epsilon_{0} D^{2} r\right) e^{-r / D}=\left(n_{0} e^{2} / 2 \pi \epsilon_{0}^{2} r\right) e^{-r / D} .
$$

This shows the Debye screening effect providing a cutoff in the coulomb interaction appearing in the spatial correlation of the electric field.

The effect is shown in a different fashion in the potential fluctuations:

$$
\begin{aligned}
\overline{\phi_{K \omega}^{2}} & =\left(2 k T / \pi K^{2} \omega\right) \operatorname{Im}(1 / \epsilon) \\
\overline{\phi_{K}^{2}} & =k T / \epsilon_{0} K^{2}\left(1+K^{2} D^{2}\right) \\
\overline{\phi\left(r_{1}\right) \phi\left(r_{2}\right)} & =\left(k T / 4 \pi \epsilon_{0}\right)\left(1-e^{-r / D}\right) / r .
\end{aligned}
$$

Here the potential correlation tends to a $1 / r$ behavior at distances large compared with $D$. The mean square potential fluctuation at any point is

$$
\overline{\phi^{2}}=k T / 4 \pi \epsilon_{0} D=(k T / e)^{2} / 8 \pi n_{0} D^{3} .
$$

The space charge density fluctuations are given by

$$
\begin{gathered}
\overline{\rho_{K \omega}^{2}}=\left(2 k T K^{2} \epsilon_{0}^{2} / \pi \omega\right) \operatorname{Im}(1 / \epsilon) \\
\overline{\rho_{K}^{2}}=k T \epsilon_{0} K^{2} /\left(1+K^{2} D^{2}\right) \\
\overline{\rho\left(r_{1}\right) \rho\left(r_{2}\right)}=-\left(k T \epsilon_{0} / 4 \pi D^{4}\right) e^{-r / D} / r \quad(r>0) .
\end{gathered}
$$

This expression for the spatial correlation of the space charge excludes the singularity at $r=0$ which corresponds to the coexistence of a charge at $r_{2}$ if one is located to $r_{1}$. The correlation function is negative due to the character of the coulomb interaction.

If $n$ and $N$ are the local densities of electrons and ions we can write (3.16) in the form

$\overline{\left(N_{1}-n_{1}\right)\left(N_{2}-n_{2}\right)}=\overline{\rho_{1} \rho_{2}} / e^{2}=-n_{0} e^{-r / D} / 2 \pi r D^{2} \quad(r>0)$.

Since the contributions of the electron-electron, electron-ion and ion-ion interactions are dependent only on charge and not on mass we have for the individual spatial correlation of the density fluctuations

$\overline{\Delta N_{1} \Delta N_{2}}=\overline{\Delta n_{1} \Delta n_{2}}=-\overline{\Delta n_{1} \Delta N_{2}}=-n_{0} e^{-r / D} / 8 \pi r D^{2}(r>0)$.

This result gives the pair correlation in an electronion plasma compatible with the Debye-Hückel theory in its usual linearized form.

The mean coulomb interaction energy per unit volume of the plasma can now be found from these pair correlations:

$$
\left.U_{\text {coul }}=\frac{1}{2} \int_{0}^{\infty} \overline{\left(\rho_{1} \rho_{2}\right.} / 4 \pi \epsilon_{0} r\right) 4 \pi r^{2} d r=-n_{0} e^{2} / 4 \pi \epsilon_{0} D .
$$

The kinetic energy density of the electrons and ions has a mean value of

$$
U_{\text {kin }}=3 k T n_{0} .
$$

The ratio of the magnitudes of the coulomb and kinetic energy densities is

$$
\left|U_{\text {coul }}\right| / U_{\text {kin }}=1 / 24 \pi n_{0} D^{3} .
$$

For the plasma to behave like a perfect gas, this ratio must be small which requires that $n_{0} D^{3}$ be larger than unity. Since $D$ is proportional to $n_{0}^{1 / 2}$ this imposes an upper bound on the required electron density. An equivalent condition is obtained by demanding that the RMS potential fluctuation shall be small compared with $k T / e$ which from (3.13) also requires that $n_{0} D^{3}$ be larger than unity. Furthermore it is needed for the validity of linearizing the Poisson- 
Boltzmann equations in the Debye-Hückel theory. Summing up, therefore, we may say that the present discussion of coulomb interaction in the longitudinal mode is applicable only if

$$
n_{0}<\left(k T \epsilon_{0} / 2 e^{2}\right)^{3} .
$$

\section{Equilibrium Plasma With Collisions}

\subsection{Current Fluctuation Spectrum}

Consideration will now be given to an equilibrium plasma with electron collision frequency $\nu$ which, for generality of presenting the comparison with the collisionless case, will be assumed to be an arbitrary function of speed.

The permittivity (to which the ionic contribution is neglected) is found from the Boltzmann equation approach to be

$$
\epsilon(\omega)=\epsilon_{0}+\frac{e^{2}}{3 m} \int_{0}^{\infty} \frac{\partial}{\partial v}\left[\frac{v^{3}}{i \omega(i \omega+\nu)}\right] 4 \pi C f_{0}(v) d v .
$$

Here $f_{0}(v)$ is the electron state occupation function and $C=2 m^{3} / h^{3}$ with the electron density given by the integrated occupancy over all phase space

$$
n_{0}=\int_{0}^{\infty} 4 \pi C v^{2} f_{0}(v) d v \text {. }
$$

Now by the fluctuation-dissipation theorem

$$
\begin{gathered}
\overline{J_{K \omega}^{2}}=(2 k T / \pi)(-\omega \operatorname{Im} \epsilon) \\
=\frac{2 e^{2} k T}{3 \pi m} \int_{0}^{\infty} \frac{\partial}{\partial v}\left(\frac{\nu v^{3}}{\nu^{2}+\omega^{2}}\right) 4 \pi C f_{0}(v) d v
\end{gathered}
$$

for any one component of the current density. Integration over frequency gives the sum rule.

$$
\overline{J_{K}^{2}}=n_{0} e^{2} k T / m=k T \epsilon_{0} \omega_{p}^{2}
$$

which applies for any damping mechanism with any velocity dependence of the collisional relaxation time.

The connection between the Boltzmann equation expression for the imaginary part of the permittivity and the current fluctuations is now made without appealing to the fluctuation-dissipation theorem (4.2) by using statistical microscopic arguments. At this point the temperature does not appear and in fact it can only manifest itself when the statement that $f_{0}$ is a thermal equilibrium distribution is inserted.

We start by noting that in a volume $V$ the variance of the number of electrons $N_{v}$ in the velocity interval $(v, v+\boldsymbol{d} v)$ is

$$
\operatorname{var} N_{v}=4 \pi C f_{o}\left(1-f_{0}\right) V v^{2} \cdot d v
$$

using the occupation fluctuation for Fermi particles.
The autocovariance of $N_{v}$ is determined by the momentum relaxation time:

$$
\operatorname{cov}\left[N_{v}\left(t_{0}\right) N_{v}\left(t_{o}+t\right)\right]=\left(\operatorname{var} N_{v}\right) \exp [-\nu(v) t] .
$$

The $v$-class current-density $K$-spectrum has the autocovariance

$$
\overline{J_{v}\left(t_{0}\right) J_{v}\left(t_{0}+t\right)_{K}}=\left(e^{2} v^{2} / 3 V\right) \operatorname{cov}\left[N_{v}\left(t_{0}\right) N_{v}\left(t_{0}+t\right)\right]
$$

where the factor $1 / 3$ arises from considering a single component of $J$.

Integrating over velocities gives

$$
\overline{J\left(t_{0}\right) J\left(t_{0}+t\right)_{K}}=\frac{e^{2}}{3} \int_{0}^{\infty} 4 \pi C f_{0}\left(1-f_{0}\right) v^{4} e^{-\nu t} d v
$$

Using the cosine transform to give the frequency spectrum yields

$$
\overline{J_{K \omega}^{2}}=\frac{2 e^{2}}{3 \pi} \int_{0}^{\infty} \frac{\nu}{\nu^{2}+\omega^{2}} 4 \pi C f_{0}\left(1-f_{0}\right) v^{4} d v
$$

Now for electrons in equilibrium the occupation function $f_{0}$ is the Fermi-Dirac function

$$
f_{0}=\left[\exp \left(\frac{1}{2} m v^{2}-\mu\right) / k T+1\right]^{-1}
$$

where $\mu$ is the Fermi energy or chemical potential. We then have the identity.

$$
\frac{\partial f_{0}}{\partial v}=-\frac{m v}{k T} f_{0}\left(1-f_{0}\right)
$$

so that the current density fluctuations may be written

$$
\begin{aligned}
\overline{J_{K \omega}^{2}}=-\frac{2 e^{2} k T}{3 \pi m} \int_{0}^{\infty} \frac{\nu}{\nu^{2}+\omega^{2}} 4 \pi C \frac{\partial f_{0}}{\partial v} v^{3} d v \\
\quad=\frac{2 e^{2} k T}{3 \pi m} \int_{0}^{\infty} \frac{\partial}{\partial v}\left(\frac{\nu v^{3}}{\nu^{2}+\omega^{2}}\right) 4 \pi C f_{0} d v .
\end{aligned}
$$

Thus the statistical approach and the thermodynamic result (4.3) agree as they must. The fluctuation temperature of the plasma and the kinetic temperature of the electrons are therefore identical and this identity has been demonstrated for any arbitrary dependence of $\nu$ on $v$ and for Fermi-Dirac statistics. One can go further and consider a charged boson gas and the same result (4.9) is attained; to do this the +1 is changed to -1 in $(4.7)$ and $\left(1-f_{0}\right)$ is replaced in (4.8) by $\left(1+f_{0}\right)$.

Statements which have appeared in the literature that the fluctuation and electron temperatures agree only under such restrictive conditions as a Maxwellian distribution or a velocity-independent relaxation time are seen to be erroneous. 
5. Appendix A. Sum Rules and Spectrum Integrals

$$
\begin{aligned}
& \int_{0}^{\infty} \frac{d \omega}{\omega} \operatorname{Im} \frac{1}{\epsilon(K, \omega)}=\frac{\pi}{2}\left[\frac{1}{\epsilon(K, \infty)}-\frac{1}{\epsilon(K, 0)}\right] \\
& \int_{0}^{\infty} \frac{d \omega}{\omega} \operatorname{Im} \frac{1}{\epsilon(K, \omega)-K^{2} / \omega^{2} \mu_{0}}=\frac{\pi}{2} \frac{1}{\epsilon(K, \infty)} \\
& \int_{0}^{\infty} \frac{d \omega}{\omega^{3}} \operatorname{Im} \frac{1}{\epsilon(K, \omega)-K^{2} / \omega^{2} \mu_{0}}=\frac{\pi}{2} \frac{\mu_{0}}{K^{2}} \\
& \int_{0}^{\infty} d \omega(-\omega \operatorname{Im} \epsilon)=(\pi / 2) \epsilon_{0}\left(\omega_{p}^{2}+\Omega_{p}^{2}\right) \quad(\mathrm{A}-4) \\
& \int_{0}^{\infty} \frac{K \sin K r}{K^{2}+A^{2}} d K=\frac{\pi}{2} e^{-r A} \quad(r>0) \quad(\mathrm{A}-5) \\
& \int_{0}^{\infty} \frac{K^{3} \sin K r}{K^{2}+A^{2}} d K=-\frac{\pi}{2} A^{2} e^{-r A} \quad(r>0) \\
& \int_{0}^{\infty} \frac{K \sin K r}{\left(K^{2}+A^{2}\right)\left(K^{2}+B^{2}\right)} d K=\frac{\pi}{2} \frac{e^{-A r}-e^{-B r}}{B^{2}-A^{2}} \quad(r>0) \\
& \int_{0}^{\infty} \frac{K^{3} \sin K r}{\left(K^{2}+A^{2}\right)\left(K^{2}+B^{2}\right)} d K=\frac{\pi}{2} \frac{B^{2} e^{-B r}-A^{2} e^{-A r}}{B^{2}-A^{2}} \quad(r>0) .
\end{aligned}
$$$$
\int_{0}^{\infty} \frac{\sin K r}{K\left(K^{2}+A^{2}\right)} d K=\frac{\pi}{2} \frac{1-e^{-r A}}{A^{2}} \quad(r \geqslant 0)
$$ 